\title{
Per l'identificazione del «Gaufredo» citato dal Marqués de Santillana
}

\author{
Towards the identification of the «Gaufredo» \\ mentioned by the Marqués de Santillana \\ Paolo Cherchi \\ (University of Chicago, Università di Ferrara)
}

\section{SOMMARIO}

Nella Defunsión de Don Enrique de Villena, testo del Marqués de Santillana, appare citato un certo «Gaufredo» che non si è potuto identificare chiaramente finora. Le proposte d'idenficazione sono scarsamente credibili semplicemente perché i nomi ricordati non ebbero mai una risonanza che autorizzasse la loro presenza in un canone. Si propone Gaufredo di Viterbo, autore del Pantheon, opera legata alla cultura spagnola. I lettori del Marqués avrebbero riconosciuto immediatamente questo scrittore.

\section{Parole chiave}

Gaufredo di Viterbo, Marqués de Santillana.

\begin{abstract}
In the Defunsión de Don Enrique de Villena by the Marqués de Santillana, the name of a certain «Gaufredo» appears that has not been clearly identified so far. The proposals of the critics for identification are scarcely credible since the suggested names never had a resonance in the Middle Ages that might authorize their presence in the literary canon. In this article the name proposed for this character is Gaufredo di Viterbo, author of the Pantheon, a work related to the Spanish culture. Readers of the Marqués de Santillana would have recognized this writer immediately.
\end{abstract}

\section{KEYWORDS}

Gaufredo di Viterbo, Marqués de Santillana.

Rebut: 01/03/2020

Acceptat: 04/05/2020 
Nell'edizione più recente della Defunsión de Don Enrique de Villena, señor docto e de exçellente ingenio, curata da Regula Rohland de Langben (Santillana, 1997: 122-132), si leggono le seguenti ottave:

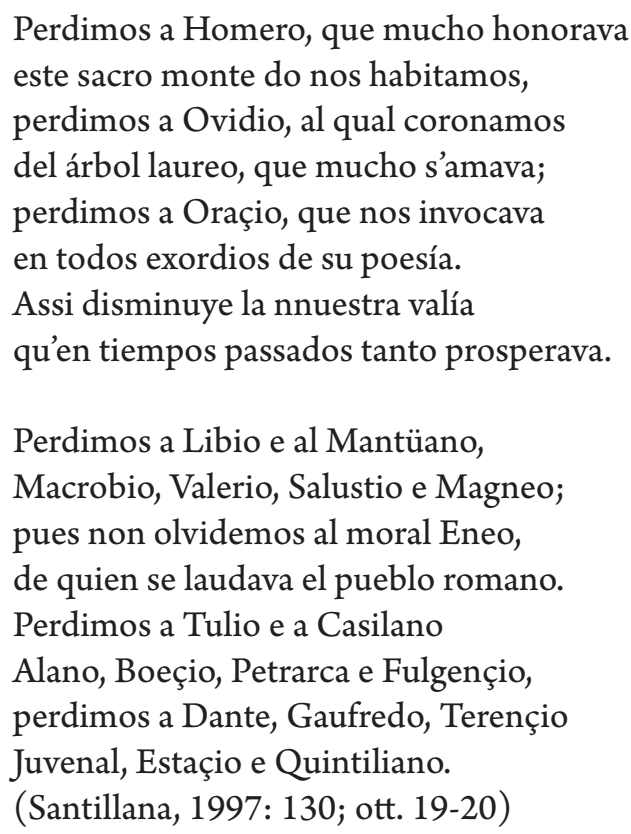

Sono ottave di grande interesse per il canone che delineano e compongono quella che potremmo chiamare «la bella scuola» del Marqués de Santillana e in genere di una nuova generazione della cultura castigliana che si sta aprendo sempre più alle correnti umanistiche ${ }^{1}$. È un canone su cui si potrebbero fare numerose osservazioni a cominciare dagli accostamenti che forse non intendono avere alcun significato. Ad esempio, richiederebbe una sottigliezza notevole capire perché Omero nella prima ottava (è anche l'unico autore greco) sia collocato vicino a Ovidio e a Orazio; oppure come nell'ottava successiva Dante appaia a fianco di Terenzio e del non identificato Gaufredo, o perché Stazio appaia in compagnia di Giovenale e Quintiliano. Probabilmente gli accoppiamenti non furono pensati con il criterio di stabilire un canone e la collocazione avrà semplicemente ragioni metriche. Forse il Marqués intendeva creare soltanto una galleria di letterati insigni nei quali fosse possibile vedere i maestri ideali del defunto Enrique de Villena, e che nello stesso tempo ne rispecchiasse la versatile e disparata attività letteraria.

Ma la costruzione del canone, se mai il Marqués de Santillana intese consegnarlo a queste menzioni, non è il motivo per cui ci soffermiamo sulle ottave riportate: il discorso sul canone richiederebbe ben altro impegno dal momento che spazia in un campo culturale vastissimo. Il nostro proposito è molto più limitato, addirittura minuscolo in quanto ci interessa semplicemente proporre l'identificazione di un autore sul quale i commentatori non si pronunciano con sicurezza. Tutti gli autori ricordati, infatti, sono agevolmente identificabili, incluso il Magneo dietro il quale senza un minimo sforzo si riconosce Lucano, il cantore della Pharsalia in cui si narra delle gesta di Pompeo Magno; e così dietro Casillano vediamo agevolmente Johannes Cassianus, autore delle Constitutiones che ebbero una notevole circolazione. L'unico autore che rimanga non 
identificato con certezza è il «Gaufredo» collocato fra Dante e Terenzio, senza, però, che in questa collocazione, come abbiamo già detto, sia da cercare un significato.

Il primo ad identificarlo con Jaufre Rudel fu Amador de los Rios che fu anche il primo editore dell'opera di Santillana. Vale la pena trascrivere per intero il testo in cui avanza la candidatura del trovatore, e si vedrà — contrariamente a quanto si desume dalle menzioni dei commentatori più recenti, i quali lo riportano in modo parziale e inesatto- che la proposta è fatta con cautela e anzi con il suggerimento che dietro quel nome «Gaufredo» sia da vedere un cronista e poeta spagnolo. Ricordiamo che l'identificazione non ha luogo nelle note al testo, bensì nell'indice dei nomi:

Gaufredo. Acaso alude don Iñigo, cuando escribe este nombre en su composicion á la muerte de don Enrique de Aragón, al celebrado Jofre o Godofre de Rodel, uno de los primeros poetas provenzales, más famoso aun por sus empresas amorosas que por sus versos. Segun el jesuita Quadrio murió en 1162, enamorado de la condesa de Trípoli. Mas también pudiera sospecharse, y esto con bastante fundamento, que se refiere el marqués á Jofre García de Loaysa, arcediano de Toledo que floreció durante los reinados de San Fernando y su hijo don Alonso, y que además de haber escrito una Chrónica de España en lengua castellana, puede no sin razon ser tenido por el autor del Poema de Alexandre, cuyo original, que poseía el marqués, se custodia en la bibl. de Osuna (Plut. III, lit. M., núm. 18). Mas dejando aparte esta cuestión para su propio lugar, en la Historia de la literatura española que hace años escribimos, bástenos lo apuntado, respecto de este autor, sin que la inseguridad de la cita nos consienta hacer otras observaciones (Amador de los Ríos, 1852: 617).

Come si vede, l'identificazione non è data per certa, anzi Amador ne propone un'altra che a suo parere avrebbe migliori credenziali. È interessante l'osservazione sul motivo della fama di Jaufre Rudel: non sono i suoi versi ma la leggenda del suo amore per la contessa di Tripoli, il suo amors de lonh. La conseguenza è chiara: il trovatore non avrebbe i titoli sufficienti e comunque pertinenti per entrare a far parte di un canone letterario e di stare a fianco di autori di grande rilevanza nel campo delle lettere. E se mai Santillana avesse voluto includere un trovatore nella sua galleria, avrebbe quasi certamente scelto Arnaut Daniel che, tra l'altro, nomina, benché di seconda mano, come poeta provenzale nel suo Proemio e Carta ${ }^{2}$.

L’identificazione con Jaufre Rudel poteva sembrare ovvia, quasi spontanea, per noi moderni, e non ha neppure tentato Curtius, il grande mestro di «canoni» e della trasmissione culturale realizzata dalla scuola. Nel suo classico Europäische Literatur und lateinisches Mittelalter del 1948 ha modo di occuparsi dei versi ricordati del Marqués de Santilla e li contestualizza nella particolare cultura spagnola di matrice più «romantica» che «classica»:

Spanien weicht auch unter dem Gesichtspunkt der Kanonbildung und der Epochenbezeichnung vom übrigen Europa ab. Die spanische Literaturgeschichte weist zunächst die Besonderheit auf, dass sie eine Romantik, aber klassik verzeichnet. Noch bemerkenwerter ist, dass die keine iberischen Autoren der Keiserzeit zur Nationalliteratur gerechnet warden. Die beiden Seneca, Lucan, Martial, Quintilian, Pomponius Mela, Juvencus, Prudentius, Merobaudes, Orosius, Isidor und andere erscheinen in den

2. «Mediocre [grado] usaron aquellos que en vulgar escrivieron, asy commo Guido Janunçello, boloñés e Arnaldo Daniel, proençal. E commo quier que destos yo no he visto obra alguna, pero quieren algunos aver ellos sido los primeros que escrivieron terçio rimo e aun sonetos en romance, e asy commo dize el philósopho, de los primeros primera es la especulación» (Santillana, 1988: 444). 
verbreitsten modernen Lehrbüchern, die damit dem Gebrauch des Mittelaters und der Renaissance getreulich folgen. Der Marqués de Santillana (15. Jahrhundert) geht in seiner Poetik von Isidor und Cassiodor aus. In seiner Lechenklage auf Enrique de Villena $(+1433)$, gibt er einen Autorenkatalog, der folgende Namen umfasst: Livius, Virgil, Macrobius, Valerius Flaccus, Sallust, Seneca, Tullius, Cassalianus (?), Alan, Boethius, Petrarca, Fulgentius, Dante, Galfrid von Vinsauf, Terenz, Juvenal, Statius, Quintilian. Was bedeutet das? Santillana repräsentiert die erste Welle des Italianismus in Spanien, aber er konserviert zugleich die mittelalterliche Auffassung der auctores: alle sind gleich gut, alle sind zeit- und geschichtslos (Curtius, 1948: 270).

È verissimo che il canone spagnolo sia diverso da quello italiano: un umanista italiano coevo del Marchese non avrebbe accostato Virgilio a Fulgenzio o a Boezio. Curtius, però, cade anche in qualche imprecisione: dimentica Omero e Lucano (Manneo); e rimane perplesso rispetto a Cassaliano che, come già osservato, è Cassiano, che è perfino presente nella sua Biblioteca del Santillana ${ }^{3}$. Tuttavia ciò che a noi interessa in questa occasione è che Gaufredo venga identificato, e senza esitazione alcuna, con Geoffroi de Vinsauf.

Il suggerimento di Curtius è stato accettato, attraverso la mediazione della traduzione in inglese del 1953, da Keith Whinnom e da Charles Faulhaber, e poi della traduzione in spagnolo (1955) da Luisa López Grigera, la quale riassume ottimamente lo status questionis in una densa nota che qui trascriviamo:

Acaso sea acertado identificar al Gaufredo de la «Defunsión de don Enrique Villena» de Santillana con Gaufroi de Vinsauf, como hacen Curtius (ob. cit., pág. 376, n. 67) y Whinnom (ver n. 19), ya que sabemos que acaso Vinosalvo haya pasado por España, no tanto por la hipótesis de Langlois, como por el verso 2066 de la Poetria nova: «Jam maris trancurri, Gades in littore fixi» tal como lo advierte Charles Faulhaber. «Retóricas clásicas y medievales en la bibliotecas castellanas, en Abaco, núm. 4, pág. 199 nota 27, y sobre todo sabiendo que se conservan en bibliotecas españolas tres códices de la Poetria, los tres de fin del siglo XIV, principios del XV, y que el Breve compendium artis rhetorice de finales del siglo XIV o principio del XV, de un tal Martinus cordubensis, se inspira fuertemente en la Poetria de Gaufridus», (Ch. F, ob. cit., pág, 156 núms.s. 118 y 119, aunque se podria pensar en la posibilidad de que la alusión de Santillana se referiera a Gaufridus de Eversely, autor de un Ars epistolaris ornatus, que en el codice de Perugia (conf. Faral, pág. 115) lleva esta advertencia: «Gaufridus anglicus hoc fecit opus in laudem domini Alfonsi, illustris regis Castelle ac etiam Legionis $\gg$. Este ars dictandi es «tal vez el primero escrito en España» (Ch. F. pág. 214, nota 55), sin embargo no se conserva en códices del siglo XV. Lo que si sabemos cualquiera de ambos sea, es que Santillana en esa obra, como lo advierte Lapesa, «abandona el octosílabo, metro en que había compuesto los decires amatorios y emplea el verso de arte mayor, más adecuado a la sonoridad grandilocuente. E intensifica la elaboración retórica, lo recursos eruditos y el latinismo del lenguaje tratando así de levantar su estilo» (ob. cit., p.134). Lo que conviene agregar es que los hombres de letras. «perdidos», que recuerda Macrobio, Alano [de Insulis], Boecio, Tulio, Gaufredo [cualquiera de ambos] y Quintiliano, son retóricos ${ }^{4}$.

3. Per tale biblioteca si veda Schiff(1905); qui a «Jean Cassien» è dedicato il cap. XXVI (1905: 160-161).

4. López Grigera (1975: 299-315). La nota trascritta (numero 22) è a p. 304. I testi ai quali si rimanda in forma abbreviate sono Curtius e Whinnom (1960). 
Sembra certo che, nonostante qualche esitazione e nonostante l'insopprimibile tentazione di avanzare altre candidature, la tendenza prevalente sia quella di seguire Curtius e di identificare «Gaufredo» con l'autore inglese della Poetria. Così risulta anche dalle note dei più recenti commentatori del Marqués de Santillana.

Fra questi è Maximilian Kerkhof il quale, però, non l'accetta toto corde, e si limita ad includerla in un'ampia rosa di altre candidature:

Me parece que con más razón se pueda sostener que alude el Marqués a Jaufré de Foxa (Foixa), a quien cita en el prólogo a los Proverbios como continuador del Arte de trovar de Remon Vidal de Besaduc [...] También don Enrique de Villena cita en el mismo contexto a «Jofre de Foxa» en su Arte de trovar [...] También es posible que se refiera a «Galfrid de Vinsuaf» (Galfredus de Vinosalvo), poeta inglés del siglo XII, autor de la Nova Poetria, Villena lo menciona en su Consolatoria (Kerkhof, 1977: 90-91).

Ma lo stesso Kerkhof deve averci ripensato e rifiuta in blocco le varie proposte da lui elencate. In effetti, non si può escludere che il Marqués volesse arricchire la sua galleria con il ritratto di un autore recente di un'ars dictandi o di una poetria per onorare la memoria di Enrique de Villena, autore anche lui di un'Arte de trovar; ma il ricordo sarebbe poco lusinghiero: per un Enrique de Villena che si accompagna a un Virgilio, a un Dante a un Boezio e a un Cicerone, che cosa può offrire la compagnia di un oscuro Jaufre de Foxa, o anche del più noto, ma ancora di statura modestissima, Geoffroi de Vinsauf? Tanto è vero che Kerkhof e il suo coeditore in un'edizione successiva al nome «Gaufredo» pongono la glossa: «el único [autor] que, entre varias posibilidades, no queda claro» (Santillana, 1988: 162; , nota ai vv. 152-160).

Lo stato di incertezza persiste, e la vediamo nel commento di Regula Rohland Legenbehn all'edizione da cui abbiamo ricavato il testo in cui appare il nostro misterioso Gaufredo. Comincia, anche questa curatrice, ricordando alcune delle proposte che la precedono e poi ne aggiunge una del tutto nuova:

Gaufredo: hay varias propuestas de identificación para este nombre. Amador propone Jofre o Godofre Rudel, trovador que Santillana no menciona en Proemio e carta. Puede ser Jofre de Foxa, citado por Villena en su Arte de trovar, o Galfrid de Vinsauf (Galfredus de Vinosalvo), poeta inglés del siglo XII, cuya Poetria fue bien conocida y difundida en España; este autor es mencionado por Villena en su Consolatoria y hay un ejemplar de su obra en la biblioteca del Marqués de Santillana» (Santillana 1997: 131, n. 139).

A questa nota segue un rimando alle note sussidiarie che ricordano ancora un altro candidato: «Otra propuesta de López Grigera [1975: 304, n. 22] es que se trataría de Gaufridus de Everseley, autor de un ars dictandi, quizá el primero escrito en España» (Santillana 1997: 333).

Pérez Priego, l'ultimo ad aver curato un'edizione della Defunsión, accetta la proposta dominante e non osa più avanzare nuove identificazioni:

Gaufredo: Luisa López Grigera sugiere, como ya había propuesto Curtius, su identificación con Gaufroi de Vinsauf, cuya Poetria fue bien conocida y difundida en España; o incluso con Gaufridus de Everseley, autor de un ars dictandi, quizá el primero escrito en España. También hace notar que en este catálogo de autores abundan los retóricos, como Macrobio, Alano, Boecio, Tulio, Gaufredo y Quintiliano («Algunas precisiones sobre el estilo 
de Antonio de Guevara», en Studia hispánica in honorem R. Lapesa, III, Madrid, Gredos, 1975 , p. 304, n. 22$)^{5}$.

Come si vede, dunque, le proposte non mancano e si ripetono, e sono tutte in misura diversa scarsamente credibili per il semplice fatto che i nomi ricordati non hanno e non ebbero mai la risonanza che autorizzasse la loro presenza in un canone. Del resto la varietà delle candidature è un indice della debolezza delle stesse.

La perplessità generale è che tutti i candidati abbiano una notorietà così modesta, quando non del tutto inesistente, da poter esser identificate immediatamente: dovrebbe essere, insomma, un «Gaufredo» insigne e familiare insieme, quasi un' antonomasia per la sua stessa opera. Dovrebbe risultare strano che un autore nel complesso poco noto, venga chiamato senza altro segno di riconoscimento, magari un etnico o il nome di una sua opera. Il nome «Gaufrido» e le sue varianti era troppo generico: certo non potevano essere i Gaufrido d'Auxerre, né i Gaufredo o Geofroi de Reims per il semplice fatto che nessun dato a noi noto li pone in relazione alla Spagna. Ma quel Gaufrido poteva benissimo essere Geoffrey di Monmouth, creatore delle leggende arturiane che circolarono in Spagna. E poteva essere Gaufredo di Viterbo, noto come Godofre che per antonomasia indicava la sua opera maggiore, ossia il Pantheon così intimamente legato alla cultura spagnola. Questi, secondo noi, era proprio il Gaufredo al quale si riferisce il Marqués de Santillana senza dover aggiungere nient'altro perché era sicuro che i suoi lettori l'avrebbero riconosciuto immediatamente. E forse abbiamo veramente identificato il «Gaufredo» dietro il quale sono state indicate persone diverse!

La candidatura di Goffredo di Viterbo ha in primo luogo una base nell'affinità del nome «Gaufredo» con il nome latino «Gaufridus»; ma in questo sarebbe alla pari con gli altri nomi di Gaufridus de Everseley e di Galfredus de Vinosalvo (Galfred e Gaufred sono varianti normali del fonema Wal/Gual), ma la differenza è che il nostro Viterbensis era una figura conosciutissima in Spagna. E la ragione di tale notorietà è il suo Pantheon, una sorta di storia universale che fu un modello e una delle fonti principali nella General Historia di Alfonso X el Sabio ${ }^{6}$. Il Pantheon fu fonte anche di Juan Manuel che nel suo Libro de los estados riprese sezioni intere dall'opera del viterbensis ${ }^{7}$. Fu anche una fonte principale del Compendi historial, composto, e rimasto incompiuto, dal domenicano Jaume Domench (m. 1385) per committenza del re Pietro IV d'Aragona ${ }^{8}$. La presenza del Pantheon si intreccia alla fortuna di due leggende di grande vitalità nella cultura spagnola e testimoniate da due opere notissime quali il Libro de Alexandre e il Libro de Apolonio, quest'ultimo da consultare oggi nell'edizione curata da Manuel Alvar (1976). La fama di Goffredo di Viterbo era sicuramente ancora viva ai tempi del Marqués de Santillana, e la sua reputazione era tale da poter meritare una menzione nella galleria dei grandi autori. Purtroppo non possiamo dire che il marchese ne possedesse un esemplare nella sua biblioteca; ma questa mancanza non è determinante per il nostro argomento, visto che non vi figura neppure Stazio. Notiamo, per con-

5. Santillana (1991: 53). Le ottave riportate sono a pp. 52-53.

6. Lo studio migliore sull'argomento è quello di Materni (2009: 123-173). Si veda anche Salvo García (2016).

7. Si veda Cherchi (1985: 300-309).

8. Sull'argomento si veda Nigoi (2015: 167-178). Questo saggio e in genere il volume che lo contiene è a tutt'oggi il sussidio maggiore e migliore per lo studio della fortuna di Goffredo di Viterbo, e soprattutto del Pantheon, in Europa e particolarmente in Spagna. Notevole per la diffusione Spagnola, è l'articolo del curatore del volume, Foerster (2015: 67-87). Sorprende, però, vedere che, in tanta perfezione bibliografica presente in questo e negli altri saggi raccolti nel volume. manchino i rimandi ai lavori di Marta Materni e di Irene Salvo García da noi citati nella n. 12. 
cludere, che se il nostro Goffredo di Viterbo non è un «retorico», come dice López Grigera, era tuttavia uno storico, e in quanto tale poteva tenere compagnia agli altri storici «perdidos» come sono Livio, Sallustio e Valerio Massimo.

Ogni proposta è tale perché non ha i criteri della certezza che ne farebbero un'affermazione chiara. Ma le proposte sono più o meno buone a seconda della loro plausibilità: forse è presuntuoso ritenere che la nostra proposta sia più credibile di tutte le altre avanzate fino ad ora.

\section{Bibliografia}

Alvar, Manuel, ed. (1976), Libro de Apolonio. Estudios, ediciones, concordancias, Valencia, Fundación Juan March - Castalia, 3 vols.

Amador de los Rios, José, ed. (1852), Obras de don Iñigo Lopez de Mendoza Marqués de Santillana, Madrid, Imprenta de la calle S. Vicente, a cargo de José Rodríguez.

Cherchi, Paolo (1985), «Juan Manuel's Libro de los estados (bk. 2) and Godfrey of Viterbo», Romance Philology, 38, pp. 300-309.

Curtius, Ernst Robert (1948), Europäische Literature und lateinisches Mittelalter, Tübingen, A. Francke.

Foerster, Thomas (2015), «Twilight of the Emperor: Godofrey's Pantheon and the Hohenstaufen Inheritance in Thirteenth-Century Castile and England», in Godofrey of Viterbo and his Readers. Imperial Traditions and Universal History, ed. Thomas Foerster, London-New York, Routledge, pp. 67-87.

López GrigerA, Luisa (1975), «Algunas precisions sobre el estilo de Antonio de Guevara», in Studia hispánica in honorem R. Lapesa, Madrid, Gredos, vol. III, pp. 299-315.

Materni, Marta (2009), «"Maestre Godofré” nella Primera e Segunda Parte della General Estoria di Alfonso X el Sabio», Critica del Testo, XII, 2-3 (2009), pp. 123-173.

Nigor, Lidia (2015), «Godofrey of Viterbo and his many readers: an example from FourteenthCentury Aragon», in Godofrey of Viterbo and his Readers. Imperial Traditions and Universal History, ed. Thomas Foerster, London-New York, Routledge, pp. 167-178.

SAlvo García, Irene (2016), «Semíramis y la ciudad de Babilonia en la General estoria de Alfonso $\mathrm{X} \gg$, e-Spania: Revue interdisciplinaire d'études hispaniques médiévales et modernes, 24 June [on line].

Santillana, Marqués de (1977), Defunsión de don Enrique de Villena, señor docto e de exçellente ingenio, ed. Maximilian Kerkhof, L’Aia, Nijhoff.

, Íñigo López de Mendoza (1988), Obras completas, edición, introducción y notas de Angel Gómez Moreno y Maximilian P. A. M. Kerkhof, Barcelona, Planeta.

(1991), Poesías completas, II, ed. Miguel Ángel Pérez Priego, Madrid, Alhambra.

(1997), Comedieta de Ponza, sonetos, serranillas y otras obras, ed. Regula Rohland de Langbehn; estudio preliminar de Vicente Beltrán, Barcelona, Crítica.

Schiff, Mario (1905), La bibliothèque du Marquis de Santillana, Paris, Buoillon.

SERÉs, Guillermo (2007), «La autoridad literaria: círculos intelectuales y géneros en la Castilla del siglo XV», Bulletin Hispanique, 109, pp. 335-383.

Whinnom, Keith, ed. (1960), «Diego de San Pedro's Stylistic Reform», Bulletin of Hispanic Studies, 27, pp. 1-15. 
